Published in final edited form as:

Trends Immunol. 2014 February ; 35(2): 49-50. doi:10.1016/j.it.2013.12.001.

\title{
A New Itch to Scratch for TSLP
}

\author{
Matthew J. Turner ${ }^{1,2}$ and Baohua Zhou ${ }^{3, *}$ \\ ${ }^{1}$ Department of Dermatology, Indiana University School of Medicine, Indianapolis, IN 46202 \\ ${ }^{2}$ Richard L. Roudebush Veterans Affairs Medical Center, Indianapolis, IN 46202 \\ ${ }^{3}$ Department of Pediatrics and Department of Microbiology and Immunology, Indiana University \\ School of Medicine, Indianapolis, IN 46202
}

\begin{abstract}
Atopic dermatitis (AD) is characterized by allergic inflammation and itch. Thymic stromal lymphopoietin (TSLP) is a pro-allergic cytokine implicated in AD. A paper in Cell transforms the understanding of TSLP's functional repertoire in general and in AD in particular showing that TSLP can directly stimulate sensory neurons and provoke itch.
\end{abstract}

\begin{abstract}
Atopic dermatitis (AD) is thought to develop as a consequence of impaired skin barrier function and allergic inflammation in the skin. One of the most bothersome and even debilitating aspects of $\mathrm{AD}$ is itch (pruritus). Itch in $\mathrm{AD}$ typically begets scratching, which can in turn promote lesion formation and/or transformation to a chronic lichenified state. Thus alleviating the unpleasant sensation of itch and the disease potentiating effects of scratching are a goal of effective therapy in AD. Unfortunately, gaps in knowledge relating to mechanisms of pruritus in $\mathrm{AD}$ often leads to suboptimal results for controlling this symptom.
\end{abstract}

The pro-allergic cytokine TSLP is produced by a variety of epithelial cells, including keratinocytes, as well as dendritic cells [1]. The TSLP receptor, a heterodimer of IL-7Ra and TSLPR, is expressed on Th2 cells, dendritic cells, mast cells and type 2 innate lymphoid cells. Keratinocyte-derived TSLP expression is increased in acute and chronic lesions in patients with AD, while elevated TSLP is detected in airway epithelial cells in patients with asthma. Moreover, genetic variants in TSLP are associated with the development of AD and its most severe complications. Thus, the immunologic/inflammatory effects of TSLP in allergic diseases are an area of intense investigation [1]. A recent paper in Cell by Wilson and colleagues identifies a non-immunologic (neurostimulatory) effect of TSLP as a pruritogen, an itch-inducing stimulus [2].

Wilson et al. demonstrated expression of TSLPR and IL-7Ra transcripts in mouse and human dorsal root ganglion cells and localized TSLPR protein expression to a subset of primary afferent nerve terminals in mouse skin [2]. Injection of TSLP into mouse cheek skin induced scratching behavior in an IL-7Ra and primary afferent neuron-dependent manner. TSLPR activation of primary afferent sensory neurons required the ion channel TRPA1 but was independent of TRPV1 [2]. These data identify TSLP as a novel endogenous pruritogen

\footnotetext{
(C) 2013 Elsevier Ltd. All rights reserved.

*Corresponding author: Zhou, B. (zhoub@iu.edu).
}

Publisher's Disclaimer: This is a PDF file of an unedited manuscript that has been accepted for publication. As a service to our customers we are providing this early version of the manuscript. The manuscript will undergo copyediting, typesetting, and review of the resulting proof before it is published in its final citable form. Please note that during the production process errors may be discovered which could affect the content, and all legal disclaimers that apply to the journal pertain. 
and suggest that keratinocyte-derived TSLP may stimulate pruritus in AD and perhaps some other dermatologic conditions.

In $\mathrm{AD}$, barrier disruption impairs the function of protease inhibitors such as LEKT1 thus favoring excessive activity of endogenous serine proteases like kallikrein 5 which in turn cleave PAR2 leading to TSLP production by keratinocytes [3]. Using the aforementioned mouse cheek injection model, Wilson et. al demonstrated that tryptase, another serine protease implicated in AD triggered scratching behavior that was partially dependent on IL-7Ra and PAR2 [2]. Additional studies showed that in vitro stimulation of keratinocytes with PAR2 agonists triggered calcium influx and NFAT-dependent transcription of TSLP in a manner dependent on the ion channel; similar findings were also noted in respiratory epithelial cells [2].

To highlight the centrality of scratching in AD pathogenesis, AD is sometimes referred to as the "itch that rashes"; in other words, the idea (while an oversimplification) is that patients with $\mathrm{AD}$ scratch their pruritic skin, promoting lesion development and persistence (Figure 1A). The importance of peripheral nerves in cutaneous inflammation is highlighted by the work of Ostrowski et al. showing attenuation of psoriasiform inflammation in KC-Tie2 transgenic mice that underwent surgical axotomy of cutaneous nerves [4]. The study by Wilson et al. adds to the understanding of the function of the neuroimmune axis in the skin by showing epithelial-derived TSLP can directly stimulate primary sensory neurons, evoking robust itch/scratch behaviors in mice independent of lymphocytes and mast cells [2]. Previously, Oyoshi et. al showed experimentally-induced epidermal injury and barrier disruption by tape stripping promotes TSLP production in mouse skin [5]. Despite the strong association between TSLP and Th2 responses, studies demonstrate that TSLP induced ADlike skin phenotypes do not require lymphocytes or mast cells but rely on direct action of TSLP on skin-resident group 2 innate lymphoid cells (ILC2) ([6]; Turner, unpublished observation). Thus it is conceivable that TSLP drives the vicious itch-scratch cycle in AD as an inducer of ILC2-mediated inflammation and pruritus and as a product of scratching (Figure 1B).

Other immunomodulators may also act directly on primary sensory neurons in the skin to stimulate pruritus. These include histamine from mast cells and the Th2 cell-derived cytokine interleukin 31 (IL-31) [7]. Histamine-mediated stimulation of sensory neurons triggers release of the immunomodulatory neuropeptides substance $\mathrm{P}$ and calcitonin gene related peptide [8]. Whether TSLP or IL-31-mediated activation of sensory neurons triggers release of neuropeptides or other immunomodulatory functions of neurons remains an open question.

The proinflammatory effects of TSLP are thought to be important drivers of pathogenesis in asthma, allergic rhinitis and other allergic diseases [1]. One of the models proposed to explain the allergic march is based on the idea that keratinocytes-derived TSLP enters the systemic circulation and acts in a systemic fashion on the developing immune system and lung microenvironment predisposing children with AD to develop asthma. Local production of TSLP by the respiratory epithelia may also directly contribute to the pathogenesis of asthma and allergic rhinitis. Like the skin, the epithelium of the upper and lower respiratory tract are innervated by sensory nerves [3]. Unlike TSLP-induced AD-like phenotypes, however, TSLP-induced asthma-like airway inflammation relies on foreign antigens and $\mathrm{CD}^{+} \mathrm{T}$ cells [9]. It remains to be determined if and how sensory neurons in the respiratory tract respond to TSLP.

The discovery of TSLP as a pruritogen is transformative in that it establishes a nonimmunologic function for TSLP and suggests targeting TSLP in AD and perhaps other 
dermatologic conditions may be an effective way to treat some forms of pruritus. More broadly, the work by Wilson et al. begs the question, what else does TSLP do? Does this cytokine act elsewhere in the peripheral or central nervous or other cell populations beyond the immune system, and if so, what effector function does it elicit? What is clear is that much work remains to understand the biology of TSLP.

\section{References}

1. Zhang Y, Zhou B. Functions of thymic stromal lymphopoietin in immunity and disease. Immunologic research. 2012; 52:211-223. [PubMed: 22274860]

2. Wilson SR, et al. The epithelial cell-derived atopic dermatitis cytokine TSLP activates neurons to induce itch. Cell. 2013; 155:285-295. [PubMed: 24094650]

3. Briot A, et al. Kallikrein 5 induces atopic dermatitis-like lesions through PAR2-mediated thymic stromal lymphopoietin expression in Netherton syndrome. The Journal of experimental medicine. 2009; 206:1135-1147. [PubMed: 19414552]

4. Ostrowski SM, et al. Cutaneous denervation of psoriasiform mouse skin improves acanthosis and inflammation in a sensory neuropeptide-dependent manner. The Journal of investigative dermatology. 2011; 131:1530-1538. [PubMed: 21471984]

5. Oyoshi MK, et al. Mechanical injury polarizes skin dendritic cells to elicit a $\mathrm{T}(\mathrm{H}) 2$ response by inducing cutaneous thymic stromal lymphopoietin expression. The Journal of allergy and clinical immunology. 2010; 126:976-984. 984 e971-975. [PubMed: 21050944]

6. Kim BS, et al. TSLP elicits IL-33-independent innate lymphoid cell responses to promote skin inflammation. Science translational medicine. 2013; 5:170ra116.

7. Sonkoly E, et al. IL-31: a new link between T cells and pruritus in atopic skin inflammation. The Journal of allergy and clinical immunology. 2006; 117:411-417. [PubMed: 16461142]

8. Peters EM, et al. Neuropeptide control mechanisms in cutaneous biology: physiological and clinical significance. The Journal of investigative dermatology. 2006; 126:1937-1947. [PubMed: 16912691]

9. Headley MB, et al. TSLP conditions the lung immune environment for the generation of pathogenic innate and antigen-specific adaptive immune responses. Journal of immunology. 2009; 182:16411647. 


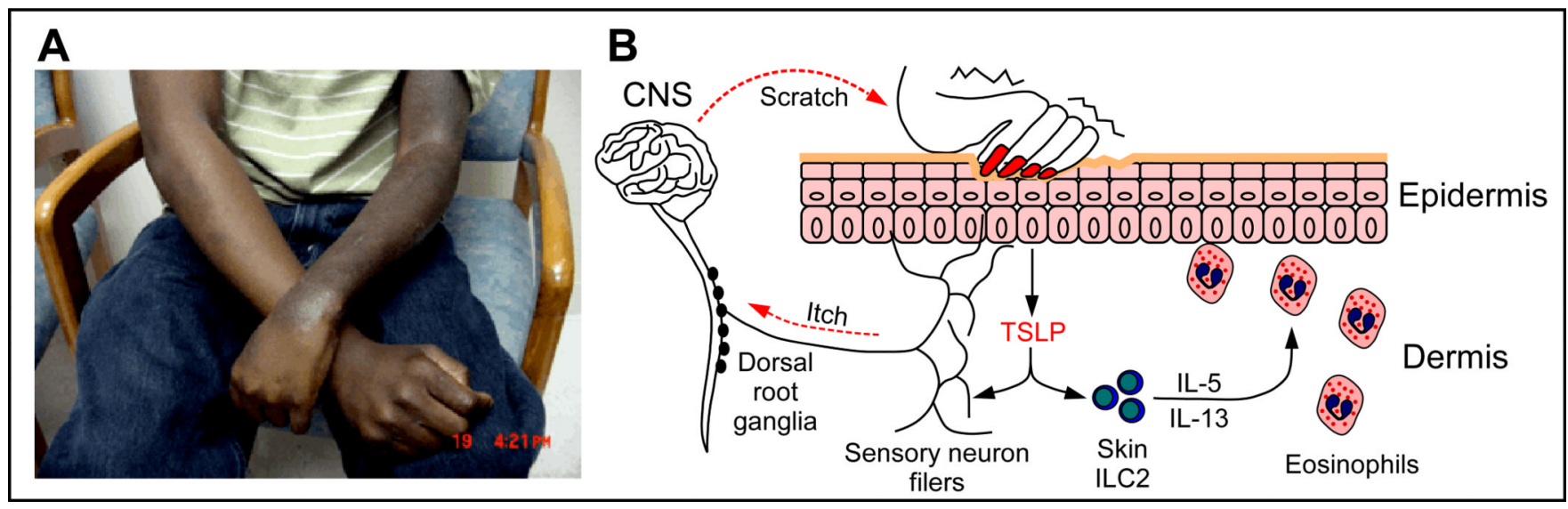

Figure 1. TSLP bridging itch/scratch and AD progression

(A) Patients with AD scratch their pruritic skin promoting lesion formation and persistence. This patient had extensive chronic lichenified AD of his left arm but relative sparing of the right arm. These findings were attributed to the patient having left-sided hemiparesis preventing him from scratching his right arm with his left hand, while being able to scratch his left arm with his normally functioning right arm. Photo courtesy of Dr. Jeffrey B. Travers, Department of Dermatology, Indiana University School of Medicine. (B) TSLPinduced itch/scratch drives AD phenotypes without the involvement of lymphocytes or mast cells. Scratch-caused epidermal injury and barrier disruption induce TSLP expression in keratinocytes. TSLP stimulates directly the sensory neurons triggering itch and subsequently, scratching behavior, and acts on skin-resident group 2 innate lymphoid cells (ILC2) to induce IL-5 and IL-13 expression leading to eosinophil recruitment and inflammation. 